\title{
新型靛玉红衍生物的合成及其对急性髓系白血病 HL-60 细胞增殖、 周期和凋亡的影响
}

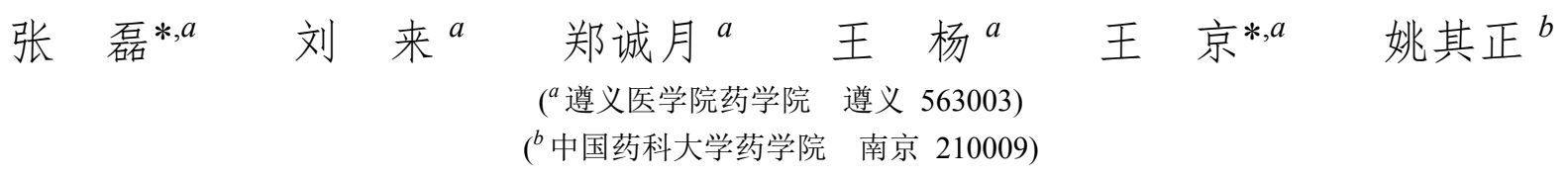

\begin{abstract}
摘要 急性髓细胞白血病是一种血液系统的恶性疾病, 严重危害人类生命健康, 目前尚无低毒、高效的治疗药物. 靛玉 红是我国传统中药青黛的有效成分，具有一定的抗白血病活性，但其水溶性差及造成的生物利用度较低，严重影响其 临床应用. 为了提高靛玉红的水溶性，进一步增强其抗白血病功效，在靛玉红结构中引入亲水性的胺基侧链，设计并 合成了五个结构新颖的靛玉红衍生物，其结构经 HRMS、 ${ }^{1} \mathrm{H}$ NMR 和 ${ }^{13} \mathrm{C}$ NMR 确证. 同时，采用 CCK-8 法测试目标产 物对急性髓系白血病 HL-60 细胞的体外抑制作用. 结果表明, 四个靛玉红衍生物具有显著的抗 HL-60 细胞增殖的活性, 其中, 化合物 $N^{1}$-(2-二甲氨基乙基)靛玉红(5a)的活性最强，其 $\mathrm{IC}_{50}$ 值为 $(3.564 \pm 0.211) \mu \mathrm{mol} / \mathrm{L}$. 流式细胞术和 Hoechst 33342 染色实验显示, 化合物 5a 能够显著诱导 HL-60 细胞周期阻滞和凋亡. 此外, 化合物 5a 还能影响细胞周期和调亡 相关蛋白的表达. 综上研究表明, 化合物 $5 \mathbf{a}$ 是一个具有深入研究价值的抗白血病先导化合物.
\end{abstract}

关键词 靛玉红; 衍生物; 抗肿瘤活性; 细胞周期; 细胞调亡; HL-60 白血病细胞

\section{Synthesis of Novel Indirubin Derivatives and Their Effects on the Proliferation, Cell Cycle and Apoptosis in Acute Myeloblastic Leukemia HL-60 Cells}

\author{
Zhang, Lei ${ }^{*, a} \quad$ Liu, Lai $^{a} \quad$ Zheng, Chengyue $^{a} \quad$ Wang, Yang ${ }^{a}$ \\ Wang, Jing $*, a \quad$ Yao, Qizheng ${ }^{b}$ \\ ( ${ }^{a}$ School of Pharmacy, Zunyi Medical University, Zunyi 563003) \\ ( ${ }^{b}$ School of Pharmacy, China Pharmaceutical University, Nanjing 210009)
}

\begin{abstract}
Acute myelogenous leukemia is a malignant disease of the hemopoietic tissue, which causes great harm to human health, and there is no therapeutic drugs with low toxicity and high efficiency. Indirubin is the active constituent of the traditional chinese medicine qingdai, which has potential anti-leukemia activity. However, poor water solubility and low bioavailability have limited its clinical treatment. To improve the water solubility and anti-leukemia activity of indirubin, hydrophilic amino side chain was linked to the indirubin, and five novel indirubin derivatives were synthesized, which were identified by HRMS, ${ }^{1} \mathrm{H}$ NMR and ${ }^{13} \mathrm{C}$ NMR. Meanwhile, the effects of target molecules on the proliferation of acute myeloblastic leukemia HL-60 cells were evaluated using CCK-8 assay. The results showed that four derivatives displayed potent antiproliferative activity against HL-60 cells. Notably, $N^{1}$-(2-dimethylaminoethyl)indirubin (5a) exhibited the best anticacner activity with an $\mathrm{IC}_{50}$ value of $(3.564 \pm 0.211) \mu \mathrm{mol} / \mathrm{L}$. Flow cytometry and Hoechst 33342 staining indicated that compound 5a could significantly trigger cell cycle arrest and induce apoptosis of HL-60 cells. Finally, compound 5a could regulate the levels of cell cycle arrest- and apoptosis-related proteins. Together, these findings revealed that compound 5a maybe be a promising lead candidate for the treatment of leukemia.
\end{abstract}

\footnotetext{
* Corresponding authors. E-mail: lzhang@zmc.edu.cn; wangjing@zmc.edu.cn

Received April 12, 2017; revised April 28, 2017; published online May 10, 2017.

Project supported by the Department of Science and Technology of Guizhou Province (Nos. [2014]7557, [2014]7565), the Priming Scientific Research Foundation for Doctoral Program of Zunyi Medical University (No. F-631), the National Undergraduate Training Programs for Innovation and Entrepreneurship (No. 201510661009), the Undergraduate Training Programs for Innovation and Entrepreneurship of Zunyi Medical University (Nos. [2014]5811, [2014]2918) and the Discipline Construction Funding (Medicinal Chemistry) of Zunyi Medical University.

贵州省科技厅(Nos. 黔科合 LH 字[2014]7557, 黔科合 LH 字[2014]7565)、遵义医学院博士科研启动基金(No. F-631)、国家级大学生创新创业(No. 201510661009)、遵义医学院大学生创新创业(Nos. 院发[2014]5811, 遵医科院发[2014]2918)和遵义医学院学科建设(药物化学)资助项目.
} 
Keywords indirubin; derivatives; anticancer activity; cell cycle; cell apoptosis; HL-60 leukemia cells

急性髓细胞白血病(acute myeloid leukemia, AML) 是一种血液系统的恶性疾病 ${ }^{[1]}$, 也是成人中最常见的急 性白血病. 目前, AML 治疗的方法主要为化疗, 常见药 物有阿糖胞苷、阿需素、依托泊苷、全反式维甲酸和三 氧化二砷等. 虽然上述药物在一定程度上能够治疗 $\mathrm{AML}$, 但 AML 患者的预后较差, 10 年生存率仍低于 $5 \%{ }^{[2]}$, 此外, 临床研究显示, 化疗药物会产生较为严重 的毒副作用 ${ }^{[3]}$ 以及耐药性 ${ }^{[4]}$. 因此, 研究新颖的低毒、高 效 AML 药物十分必要.

青黛是一种常见的中药材, 为蓼科植物蓼蓝 (Polygonum tinctorium Ait) 、爵床科植物马蓝 (Baphicacanthus cusiu (Ness) Bremek)或十字花科植物蓊 蓝(Isatis indigotica Fort.)的茎叶经炮制制得的干燥粉末、 颗粒或团块, 具有清热解毒、清肝泻火和凉血止血等功 效, 主要用于治疗吐血、小儿惊㾁、热毒痛疮、斑疹、 恶疮和湿疹等病症. 现代医学研究发现, 青黛是中成药 当归龙荟丸中抗白血病的主要成分 ${ }^{[5]}$. 近年来的研究还 表明, 双吲哚类生物碱靛玉红(Indirubin, 图 1)是青黛的 有效成分 ${ }^{[6]}$, 其具有抗真菌、抗病毒、抗炎和治疗银屑 病等多种药理活性 ${ }^{[7 \sim 10]}$. 同时, 靛玉红还具有潜在的抗 肿瘤作用 ${ }^{[11,12]}$.

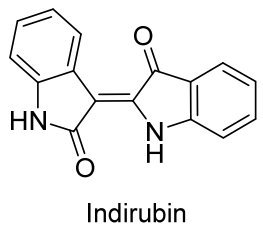

图 1 靛玉红结构

Figure 1 Structure of indirubin

虽然靛玉红显示出重要的生物活性, 但它具有一个 大 $\pi$ 共轭体系的平面刚性骨架, 因此其水溶性差, 生物 利用度低，这些缺点极大地限制其临床应用 ${ }^{[13]}$. 例如, Hsuan 等 ${ }^{[14]}$ 研究发现, 静玉红对人急性髓系白血病 $\mathrm{HL}-60$ 细胞具有一定的抑制活性, 但活性较差, $\mathrm{LC}_{50}$ 值 为 $98.1 \mu \mathrm{g} / \mathrm{L}$. 为了进一步提高静玉红的水溶性和抗肿瘤 活性, 人们对其结构进行了大量的修饰和改造工作, 如 将肜基、多元醇、糖基和胺基片段等亲水性结构引入到 靛玉红分子中, 发现了一些水溶性强及抗肿瘤活性显著 的静玉红衍生物. 例如, Hoessel 等 ${ }^{[15]}$ 发现, 3'-肜基青定玉 红(a, 图 2)能够通过阻滞细胞周期而抑制多种肿瘤细胞 株的增殖, 如人淋巴细胞瘤 Jurkat 细胞、人乳腺癌 MCF-7 细胞和人急性髓系白血病 HL-60 细胞等. 该课题 组 ${ }^{[16]}$ 又将亲水性的二醇片段连接到 6 -溴-3'-肜基靛玉红
的肜差基上，得到了化合物 $\mathbf{b}$ (图 2)，其能够显著抑制人 神经母细胞瘤 SH-SY5Y 细胞的增殖, $\mathrm{IC}_{50}$ 值为 0.94 $\mu \mathrm{mol} / \mathrm{L}$. Song 等 ${ }^{[17]}$ 研究发现, 3'-肜基责定玉红的 5-二苯乙 酰胺衍生物(c, 图 2)具有较强的抗肿瘤活性, 对多种白 血病细胞株均有较强的抑制活性, 其中对 HL-60 细胞的 抑制作用较为显著, $\mathrm{IC}_{50}$ 值为 $(1.99 \pm 0.01) \mu \mathrm{mol} / \mathrm{L}$. 分子 机制研究显示, 化合物 $\mathbf{c}$ 的抗肿瘤活性与诱导细胞凋亡

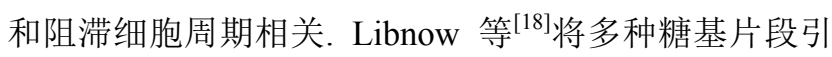
入到靛玉红的 $\mathrm{N}^{1}$ 位, 如 $L$-鼠李糖、 $D$-葡萄糖、 $D$-半乳 糖和 $D$-甘露糖. 体外抗肿瘤活性研究显示, 靛玉红的 $L$ 鼠李糖衍生物(d, 图 2)能够选择性抑制 MCF-7 细胞的增 殖, $\mathrm{IC}_{50}$ 值为 $(0.67 \pm 0.08) \mu \mathrm{mol} / \mathrm{L}$. Cheng 等 ${ }^{[19]}$ 合成了多 种靛玉红的亲水性衍生物, 其中 $N$-甲基哌嗪衍生物 $\mathbf{e}$ (图 2)具有较强的水溶性, 并且对人肺癌 LXFL529L 细 胞具有强烈的抑制活性, $\mathrm{IC}_{50}$ 值为 $0.54 \mu \mathrm{mol} / \mathrm{L}$. Wang 等 ${ }^{[20]}$ 将多种亲水性胺基侧链引入到 5-氟青定玉红分子中, 细胞毒活性结果显示, 该系列衍生物能够抑制多种肿瘤 细胞株的增殖, 其中化合物 f (图 2)对 HL-60 细胞具有较 强的抑制作用, $\mathrm{IC}_{50}$ 值为 $1.7 \mu \mathrm{mol} / \mathrm{L}$, 强于阳性药舒尼替 尼. 近年来, 笔者 ${ }^{[21 ~ 23]}$ 也报道过靛玉红-3'-肜、7-氮杂-3'肜基靛玉红以及 7,7'-氮杂(-3'-肜基)靛玉红等衍生物的 合成及其抗肿瘤活性研究.

为了提高靛玉红的水溶性, 进一步增强其抗白血病 功效, 本文在靛玉红结构中引入亲水性的胺基侧链, 设 计并合成了五个结构新颖的靛玉红衍生物, 其结构经 HRMS、 ${ }^{1} \mathrm{H}$ NMR 和 ${ }^{13} \mathrm{C}$ NMR 确证. 同时, 采用 CCK-8 法测试目标产物对 HL-60 细胞的体外抑制活性. 此外, 本文还利用流式细胞术、Hoechst 33342 染色和 Western blot 方法初步研究目标产物的作用机制. 合成路线见 Scheme 1 .

\section{1 结果与讨论}

\section{1 目标化合物的合成}

在室温条件下, 原料靛红 1 和吲哚乙酸酯 $\mathbf{2}$ 反应制 得中间体 3.3 与氯代胺基盐酸盐 4 在碳酸铯条件下制备 得到目标产物 5a、5b 和 $5 \mathrm{~d}$; 在氢化钠条件下制备得到 目标产物 $5 \mathbf{c}$ 和 $5 \mathrm{e}$, 合成路线如 Scheme 1 所示. 目标产 物的结构经 HRMS、 ${ }^{1} \mathrm{H}$ NMR 和 ${ }^{13} \mathrm{C}$ NMR 确证.

\section{2 目标化合物的体外抗肿瘤活性}

利用 CCK-8 法研究五个目标产物对 HL-60 细胞的 抗增殖活性, 靛玉红(Indirubin)和依托泊苷(Etoposide)为 阳性对照药, 活性数据如表 1 所示. 经过 $72 \mathrm{~h}$ 共同孵化 后，四个化合物显示出较强的抗肿瘤活性，如 $\mathbf{5 a} 、 \mathbf{5 b}$ 、 

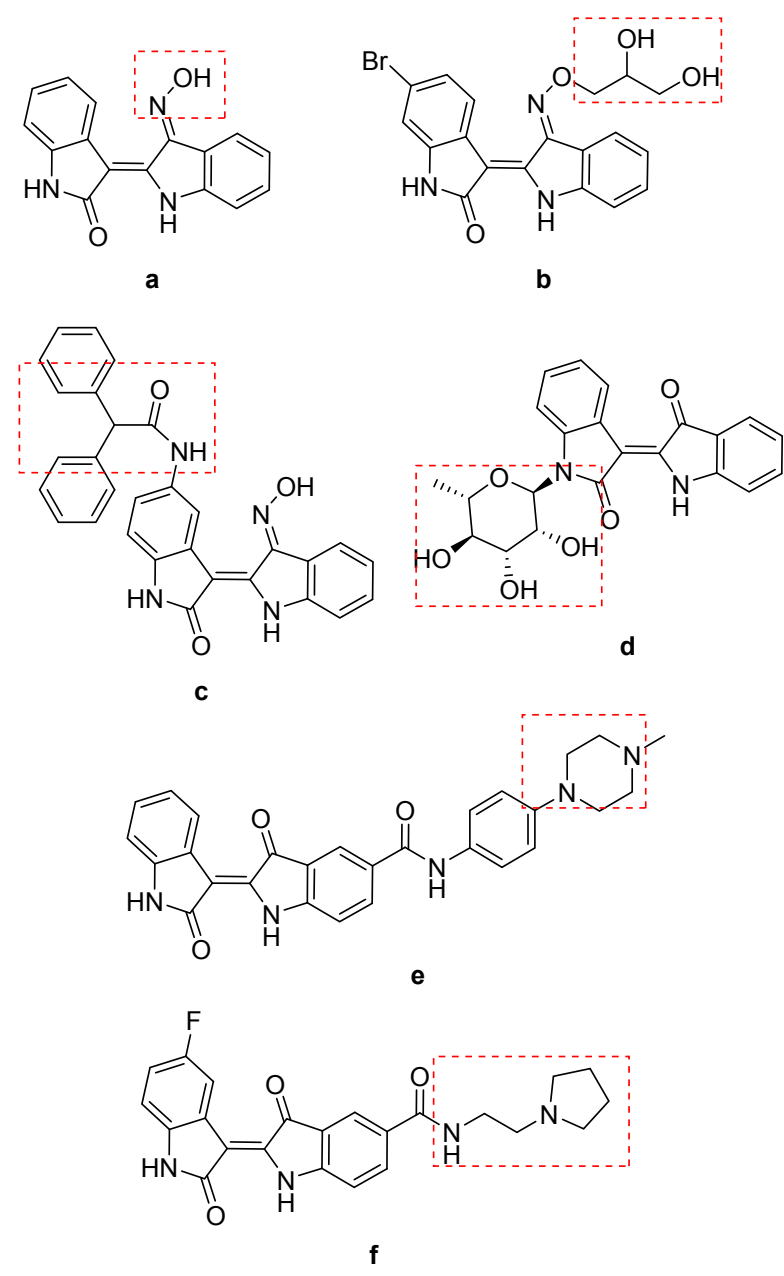

图 2 已报道的靛玉红类化合物

Figure 2 Reported indirubin derivatives<smiles>C[C+]1NC(=O)/C(=C2\Nc3ccccc3C2=O)c2ccccc21</smiles><smiles>[R]N1C(=O)/C(=C2\Nc3ccccc3C2=O)c2ccccc21</smiles>

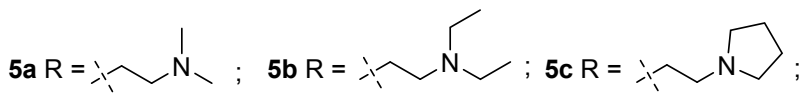

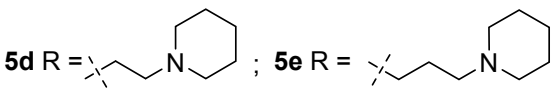

Reagents and conditions: (a) $\mathrm{Na}_{2} \mathrm{CO}_{3}, \mathrm{MeOH}, 25{ }^{\circ} \mathrm{C}$; (b) $\mathrm{Ce}_{2} \mathrm{CO}_{3}$, DMF, $25{ }^{\circ} \mathrm{C}$ for $\mathbf{5 a}, \mathbf{5 b}$ and $\mathbf{5 d}$; (c) $\mathrm{NaH}, \mathrm{DMF}, 60{ }^{\circ} \mathrm{C}$ for $\mathbf{5 c}$ and $\mathbf{5 e}$

图式 1 目标产物的合成

Scheme 1 Synthesis of target compounds

$\mathbf{5 c}$ 和 $5 \mathrm{e}$, 其 $\mathrm{IC}_{50}$ 值分别为 $(3.564 \pm 0.211) 、(11.536 \pm$
$0.44) 、(4.446 \pm 0.459)$ 和 $(8.046 \pm 0.339) \mu \mathrm{mol} / \mathrm{L}$. 而阳性 对照靛玉红没有显示出抗 HL-60 细胞增殖的作用，这可 能与靛玉红的水溶性较差有关，其 $\mathrm{IC}_{50}$ 值大于 100 $\mu \mathrm{mol} / \mathrm{L}$. 依托泊苷是一种临床上用于治疗白血病的药 物, 对 HL-60 细胞具有较强的抑制作用, $\mathrm{IC}_{50}$ 值为 $(1.163 \pm 0.025) \mu \mathrm{mol} / \mathrm{L}$. 目标产物 $\mathbf{5 a}$ 的抗肿瘤活性显著 强于母体靛玉红, 与阳性药依托泊苷相当. 上述研究表 明，在靛玉红 $\mathrm{N}^{1}$ 位引入亲水性的胺基侧链，能够不同程 度地增强化合物的抗肿瘤活性.

表 1 目标分子对 HL-60 细胞的体外细胞毒活性 Table 1 Cytotoxic effects of target molecules against HL-60 cells in vitro

\begin{tabular}{cc}
\hline Compd. & $\mathrm{IC}_{50} /\left(\mu \mathrm{mol} \cdot \mathrm{L}^{-1}\right)$ \\
\hline $\mathbf{5 a}$ & $3.564 \pm 0.211$ \\
$\mathbf{5 b}$ & $11.536 \pm 0.44$ \\
$\mathbf{5 c}$ & $4.446 \pm 0.459$ \\
$\mathbf{5 d}$ & $60.664 \pm 5.206$ \\
$\mathbf{5 e}$ & $8.046 \pm 0.339$ \\
Indirubin & $>100$ \\
Etoposide & $1.163 \pm 0.025$ \\
\hline
\end{tabular}

初步的构效关系研究显示, 将亲水性的胺基侧链连 接到靛玉红 $\mathrm{N}^{1}$ 位有利于提高化合物的抗肿瘤活性. 其 中, 引入链状胺基链时, 二甲氨基衍生物的抗肿瘤活性 优于二乙氨基; 引入环状胺基侧链时, 连接臂为两个碳 的吡咯烷和三个碳的哌啶优于两个碳的哌啶. 整体上, 引入的胺基链具有较少亲脂性原子或基团时，化合物的 抗肿瘤活性更显著，如化合物 $\mathbf{5 a}$ 和 $\mathbf{5 c}$.

\section{3 化合物 $5 a$ 对 HL-60 细胞周期的影响}

靛玉红是一种作用于细胞周期蛋白激酶的天然产 物 ${ }^{[24]}$, 为了进一步研究本文目标产物的抗肿瘤机制, 我 们利用流式细胞术检测目标产物 5a 对 HL-60 细胞周期 的影响. 如图 3 所示, 化合物 5a 与 HL-60 细胞捊化 $48 \mathrm{~h}$ 后，与对照组相比，实验组 HL-60 细胞的周期分布发生 显著变化. 在 $5 \mu \mathrm{mol} / \mathrm{L}$ 浓度下, 化合物 $5 \mathbf{a}$ 能够阻滞 $\mathrm{HL}-60$ 细胞周期在 $\mathrm{S}$ 期; 但随着浓度增加到 $10 \mu \mathrm{mol} / \mathrm{L}$, 化合物 5a 将 HL-60 细胞的周期阻滞在 G1 期. 上述实验 结果表明, 目标产物 $\mathbf{5 a}$ 能够影响 HL-60 细胞周期的分 布，并且对周期的影响与药物浓度相关.

\section{4 化合物 5a 对 HL-60 细胞凋亡的影响}

调亡调控异常在肿瘤发生、发展过程中起到关键作 用 ${ }^{[25]}$, 多种抗肿瘤药物能够通过诱导调亡的方式而产 生抗肿瘤活性 ${ }^{[26]}$. 为了探索目标产物 5a 对调亡的影响, 本文利用 Annexin V-FITC/PI 双染法研究 5a 对 HL-60 细 胞调亡的调节作用. 如图 4 所示, 作用 $48 \mathrm{~h}$ 后, 5 和 10 $\mu \mathrm{mol} / \mathrm{L}$ 的化合物 $5 \mathbf{a}$ 分别能够诱导 HL-60 细胞产生 $55.37 \%$ 和 $71.09 \%$ 的调亡率, 而对照组细胞的调亡率为 

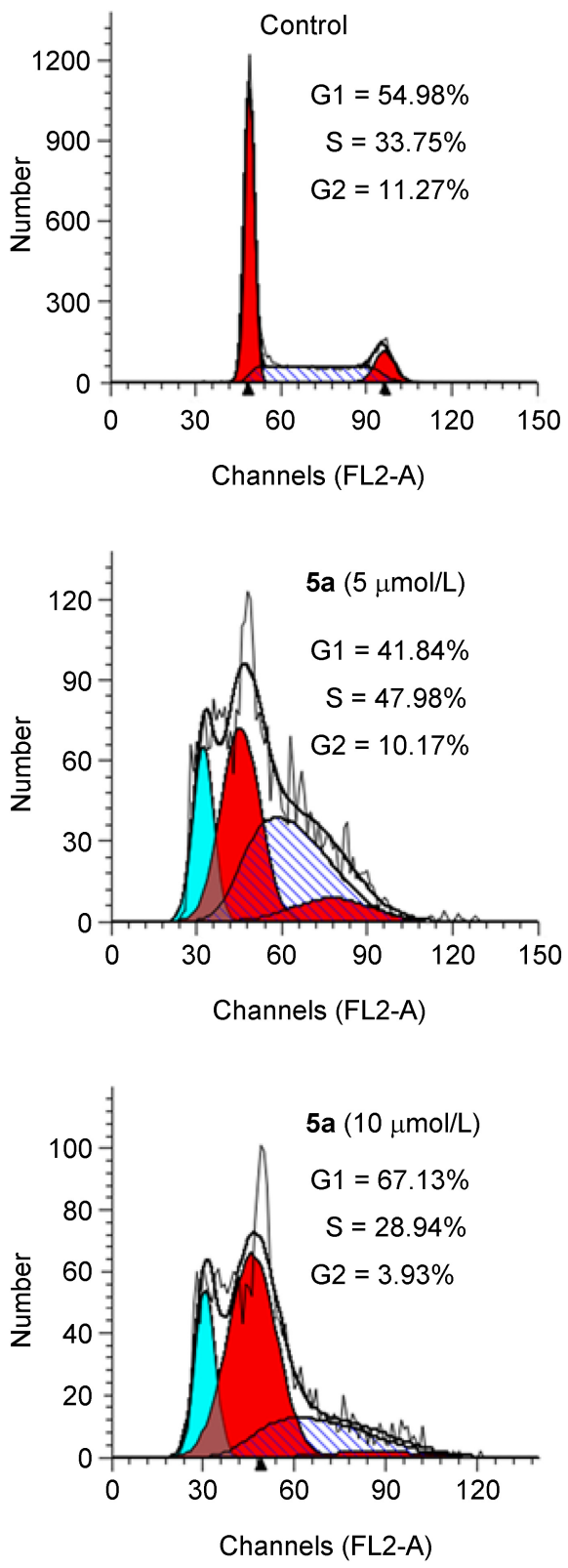

图 3 化合物 5a 对 HL-60 细胞周期的影响

Figure 3 Effects of compound $\mathbf{5 a}$ on the cell cycle of HL-60 cells

$6.65 \%$. 上述研究表明, 化合物 $5 \mathbf{a}$ 能够浓度依赖性地诱 导 HL-60 细胞调亡.

\subsection{Hoechst 33342 染色}

通过 Hoechst 33342 染色法, 进一步验证化合物 5a 对 HL-60 细胞调亡的诱导作用. 实验结果如图 5 所示, 孵化 $24 \mathrm{~h}$ 后, 化合物 5a 能够明显减少 HL-60 细胞的数 量. 同时, HL-60 细胞出现皱缩、染色质固缩、裂解和调 亡小体等现象. 并且, 化合物 $\mathbf{5 a}$ 的浓度越高, 上述凋亡 现象越显著. Hoechst 33342 染色研究进一步证明, 化合 物 5a 能够显著诱导 HL-60 细胞调亡.
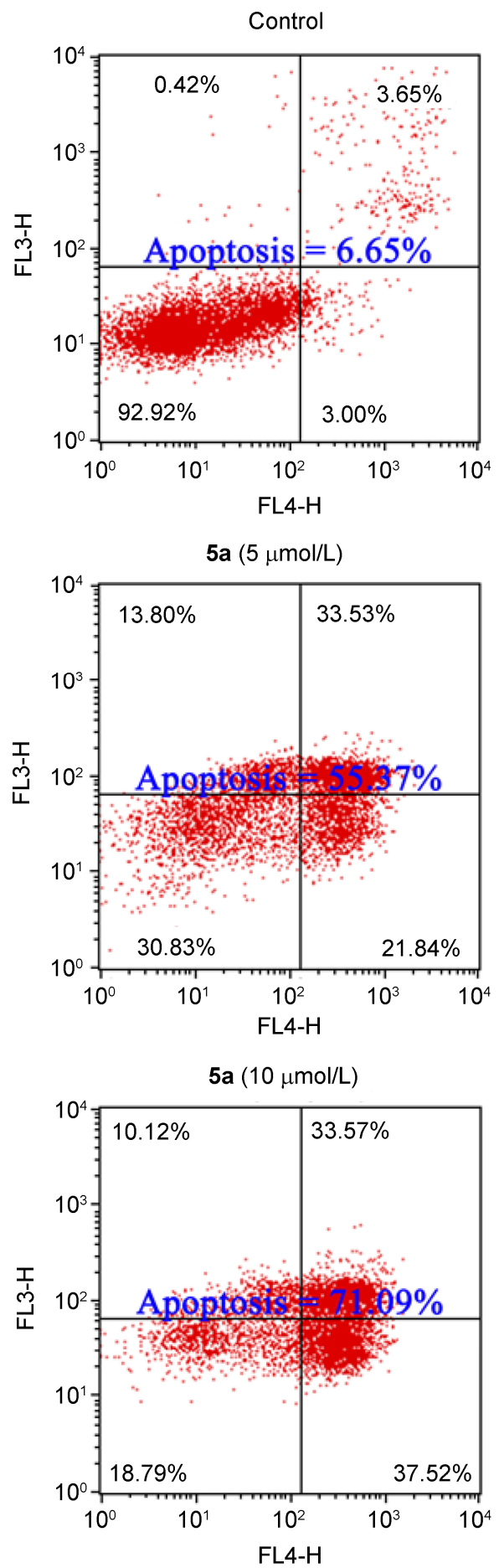

图 4 化合物 5a 对 HL-60 细胞调亡的影响

Figure 4 Effects of compound 5a on the apoptosis of HL-60 cells

\section{6 化合物 $5 a$ 对 HL-60 细胞中周期和凋亡相关蛋白表 达的影响}

在前面的研究中, 化合物 5a 能够诱导 HL-60 细胞 周期阻滞和调亡, 因此我们进一步探索化合物 5a 对 HL-60 细胞中周期和调亡相关蛋白(CDK1、CDK2 和 

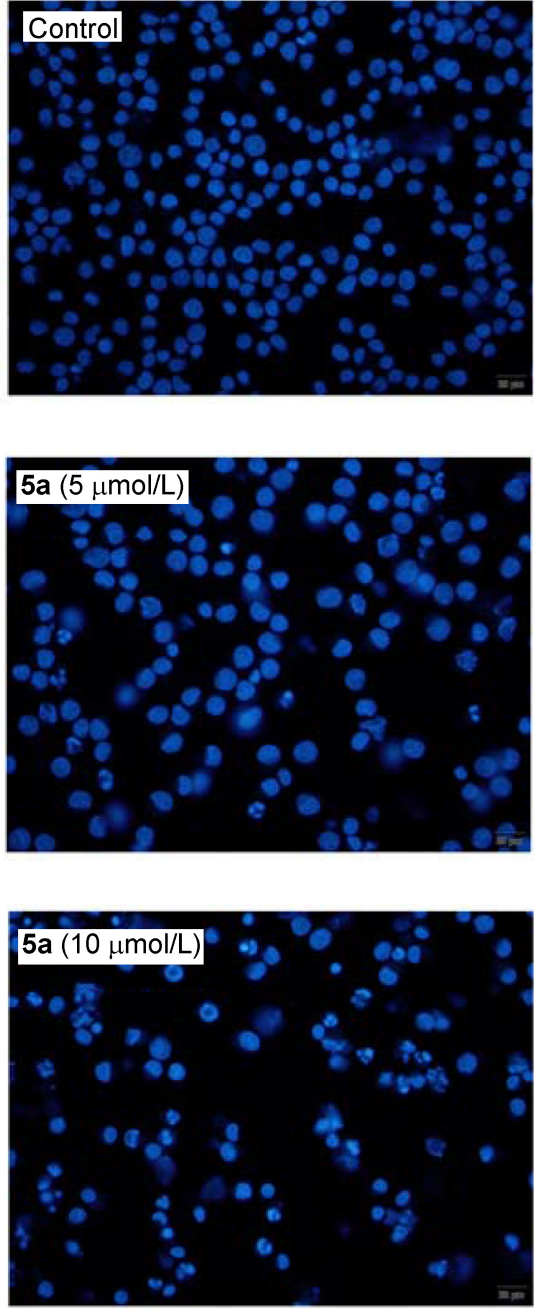

图 5 Hoechst 33342 染色

Figure 5 Hoechst 33342 staining

Cleaved Caspase-3)表达的影响. HL-60 细胞与化合物 5a 共同捊化 $48 \mathrm{~h}$ 后, 利用蛋白质免疫印迹法(Western Blot) 检测细胞中相关蛋白的表达, 结果如图 6 所示. 在 5 $\mu \mathrm{mol} / \mathrm{L}$ 浓度下, 化合物 $\mathbf{5 a}$ 对 CDK1 的表达没有影响, 但 能够下调 CDK2 的表达; 而在 $10 \mu \mathrm{mol} / \mathrm{L}$ 浓度下, 化合 物 5a 能够抑制 CDK1 和 CDK2 的的表达. 上述研究结 果说明, 化合物 5a 对 HL-60 细胞中 CDK1 和 CDK2 表 达的调控受到浓度的影响, 这也与前文的研究结果一 致, 即目标产物 5a 能够影响 HL-60 细胞周期的分布, 并 且对周期的影响与药物浓度相关.

此外, 图 6 还表明, 随着化合物 $\mathbf{5 a}$ 浓度的增加, 其 对 HL-60 细胞中 Cleaved Caspase-3 的上调作用越显著, 这也与流式细胞术检测调亡的结果一致.

\section{2 结论}

为了进一步提高天然产物靛玉红的水溶性和抗白 血病细胞增殖活性, 设计并合成了五个结构新颖的靛玉

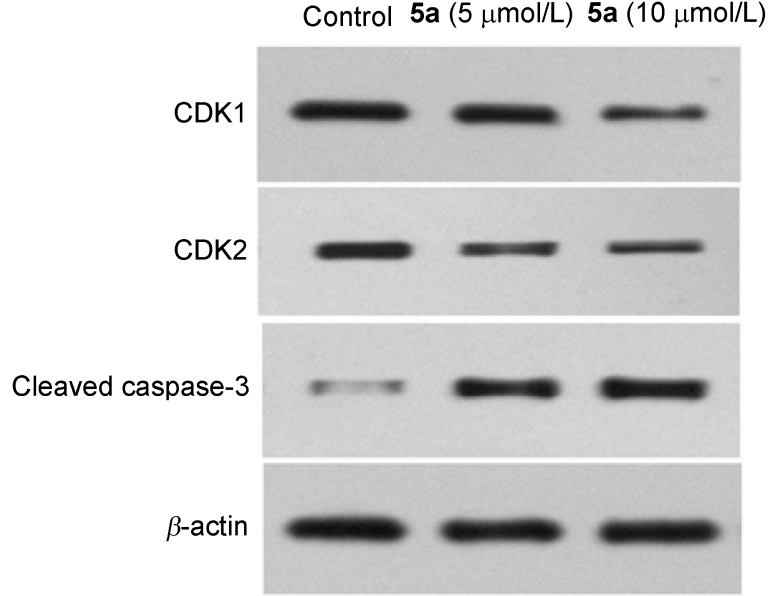

图 6 化合物 5a 对 HL-60 细胞中 CDK1, CDK2 和活化 Caspase-3 表达的影响

Figure 6 Effects of compound $\mathbf{5 a}$ on the expressions of CDK1, CDK2 and Cleaved Caspase-3 in HL-60 cells

红衍生物. CCK-8 法实验结果显示, 四个目标产物在体 外能够有效抑制 HL-60 细胞的增殖, 强于母体靛玉红. 其中, 化合物 $5 \mathbf{a}$ 的抗肿瘤活性最为显著, $\mathrm{IC}_{50}$ 值为 $(3.564 \pm 0.211) \mu \mathrm{mol} / \mathrm{L}$. 初步的分子机制研究表明，通 过调节周期和调亡相关蛋白的表达，化合物 5a 能够显 著诱导 HL-60 细胞周期阻滞和调亡，从而发挥抗肿瘤活 性.

\section{3 实验部分}

\section{1 仪器与试剂}

核磁共振仪为 Agilent-NMR-vnmrs 400 型 $\left(\mathrm{CDCl}_{3}\right.$ 为 溶剂), 质谱仪为 AgilentAccurate-Mass-Q-TOF-MS 6520 型(HRMS), 熔点为 SGWX-4 显微熔点仪. 薄层层析板 和 $200 \sim 300$ 目硅胶购自青岛海洋化工厂，所用试剂为 分析纯.

\section{2 实验方法}

中间体 3 的合成参照文献[27].

\subsection{1目标产物 $5 \mathbf{a}, \mathbf{5 b}$ 和 $5 d$ 的合成}

将 $0.38 \mathrm{mmoL}$ 中间体 $\mathbf{3}, 0.44 \mathrm{mmoL} \mathrm{4}, 0.95 \mathrm{mmoL}$ 碳酸铯加入到 $10 \mathrm{~mL}$ 圆底烧瓶中, 加入 $5 \mathrm{~mL} \mathrm{N,N-二甲}$ 基甲酰胺(DMF)溶解, $25{ }^{\circ} \mathrm{C}$ 下摚拌 $4 \sim 5 \mathrm{~h}$. 薄层色谱 (TLC)监测反应完全后, 加入 $40 \mathrm{~mL}$ 水淬灭反应, 二氯 甲烷萃取三次, 水洗两次, 饱和氯化钠溶液洗涤一次, 无水硫酸镁干燥过夜. 抽滤, 减压除去溶剂, 得到的粗 品用 $200 \sim 300$ 目硅胶柱层析[洗脱剂: $V$ (二氯甲烷)： $V$ (甲醇 $)=50: 1$ ]纯化, 得到目标产物 $5 \mathbf{a} 、 5 \mathbf{b}$ 和 $5 \mathbf{d}$.

$N^{1}$-(2-二甲氨基乙基)靛玉红(5a): 紫色固体, 产率 64\%. m.p. $167 \sim 169{ }^{\circ} \mathrm{C} ;{ }^{1} \mathrm{H}$ NMR $\left(\mathrm{CDCl}_{3}, 400 \mathrm{MHz}\right) \delta$ : 
$10.52(\mathrm{~s}, 1 \mathrm{H}), 8.86(\mathrm{~d}, J=6.0 \mathrm{~Hz}, 1 \mathrm{H}), 7.71(\mathrm{~d}, J=5.6 \mathrm{~Hz}$, $1 \mathrm{H}), 7.47$ (s, 1H), 7.28 (d, $J=6.4 \mathrm{~Hz}, 1 \mathrm{H}), 7.10(\mathrm{~s}, 1 \mathrm{H})$, $6.98 \sim 6.89(\mathrm{~m}, 3 \mathrm{H}), 3.92(\mathrm{~s}, 2 \mathrm{H}), 2.60(\mathrm{~s}, 2 \mathrm{H}), 2.34(\mathrm{~s}$, $6 \mathrm{H}) ;{ }^{13} \mathrm{C} \mathrm{NMR}\left(\mathrm{CDCl}_{3}, 100 \mathrm{MHz}\right) \delta: 188.22,170.55$, $151.57,141.12,139.26,136.84,129.11,125.59,125.20$, $122.56,121.53,121.26,120.00,111.82,107.91,106.49$, $56.46,45.67,38.12$; HRMS calcd for $\mathrm{C}_{20} \mathrm{H}_{20} \mathrm{~N}_{3} \mathrm{O}_{2}[\mathrm{M}+$ $\mathrm{H}]^{+}$334.1550, found 334.1550.

$N^{1}$-(2-乙氨基乙基)静玉红(5b)：紫色固体，产率 57\%. m.p. 119 121 ${ }^{\circ} \mathrm{C} ;{ }^{1} \mathrm{H}$ NMR $\left(\mathrm{CDCl}_{3}, 400 \mathrm{MHz}\right) \delta$ : 10.52 (s, 1H), 8.86 (d, $J=6.8 \mathrm{~Hz}, 1 \mathrm{H}), 7.70$ (d, $J=6.0 \mathrm{~Hz}$, 1H), 7.46 (s, 1H), 7.28 (d, J=6.8 Hz, 1H), 7.09 (s, 1H), $6.97 \sim 6.88(\mathrm{~m}, 3 \mathrm{H}), 3.89(\mathrm{~s}, 2 \mathrm{H}), 2.73(\mathrm{~s}, 2 \mathrm{H}), 2.64(\mathrm{~d}, J=$ $6.4 \mathrm{~Hz}, 4 \mathrm{H}), 1.05(\mathrm{~s}, 6 \mathrm{H}) ;{ }^{13} \mathrm{C} \mathrm{NMR}\left(\mathrm{CDCl}_{3}, 100 \mathrm{MHz}\right) \delta$ : $188.22,170.51,151.58,141.27,139.20,136.82,129.11$, $125.55,125.18,122.49,121.50,121.21,120.01,111.81$, 107.95, 106.54, 49.95, 47.54, 38.39, 12.00; HRMS calcd for $\mathrm{C}_{22} \mathrm{H}_{24} \mathrm{~N}_{3} \mathrm{O}_{2}[\mathrm{M}+\mathrm{H}]^{+}$362.1863, found 362.1865.

$N^{1}$-[2-(1-哌啶)乙基]靛玉红(5d): 紫色固体, 产率 60\%. m.p. 204 206 ${ }^{\circ} \mathrm{C}$; ${ }^{1} \mathrm{H}$ NMR $\left(\mathrm{CDCl}_{3}, 400 \mathrm{MHz}\right) \delta$ : 10.52 (s, 1H), $8.86(\mathrm{~d}, J=7.6 \mathrm{~Hz}, 1 \mathrm{H}), 7.71(\mathrm{~d}, J=7.2 \mathrm{~Hz}$, $1 \mathrm{H}), 7.47$ (t, $J=7.6 \mathrm{~Hz}, 1 \mathrm{H}), 7.28$ (t, $J=6.8 \mathrm{~Hz}, 1 \mathrm{H}), 7.10$ $(\mathrm{t}, J=7.2 \mathrm{~Hz}, 1 \mathrm{H}), 6.99 \sim 6.90(\mathrm{~m}, 3 \mathrm{H}), 3.94(\mathrm{t}, J=7.2 \mathrm{~Hz}$, $2 \mathrm{H}), 2.61(\mathrm{t}, J=6.8 \mathrm{~Hz}, 2 \mathrm{H}), 2.51(\mathrm{~s}, 4 \mathrm{H}), 1.60(\mathrm{~s}, 4 \mathrm{H})$, $1.44(\mathrm{~s}, 2 \mathrm{H}) ;{ }^{13} \mathrm{C} \mathrm{NMR}\left(\mathrm{CDCl}_{3}, 100 \mathrm{MHz}\right) \delta: 188.23$, $170.48,151.59,141.26,139.21,136.84,129.11,125.53$, $125.19,122.51,121.51,121.21,120.00,111.83,108.07$, 106.56, 56.01, 54.78, 37.63, 25.90, 24.20; HRMS calcd for $\mathrm{C}_{23} \mathrm{H}_{24} \mathrm{~N}_{3} \mathrm{O}_{2}[\mathrm{M}+\mathrm{H}]^{+}$374.1863, found 374.1862.

3.2.2 目标产物 $5 \mathrm{c}$ 和 $5 \mathrm{e}$ 的合成

将 $0.38 \mathrm{mmoL}$ 中间体 3 和 $0.44 \mathrm{mmoL} 4$ 加入到 10 $\mathrm{mL}$ 圆底烧瓶中, 分批加入 $1.9 \mathrm{mmoL}$ 氢化钠, 在 $\mathrm{N}_{2}$ 保 护下 $60{ }^{\circ} \mathrm{C}$ 搅拌反应 $2 \sim 3 \mathrm{~h}$. TLC 监测反应完全后, 加入 $40 \mathrm{~mL}$ 饱和氯化铵溶液淬灭反应, 二氯甲烷萃取三次, 水洗两次, 饱和氯化钠溶液洗涤一次, 无水硫酸美干燥 过夜. 抽滤, 减压除去溶剂, 得到的粗品用 200 300 目 硅胶柱层析 [洗脱剂: $V$ (二氯甲烷 $): V($ 甲醇 $)=50: 1$ ] 纯 化，得到目标产物 $5 \mathbf{c}$ 和 $5 \mathbf{e}$.

$N^{1}$-[2-(1-吡咯烷)乙基]靛玉红(5c)：紫色固体, 产率 69\%. m.p. 181 182 ${ }^{\circ} \mathrm{C} ;{ }^{1} \mathrm{H}$ NMR $\left(\mathrm{CDCl}_{3}, 400 \mathrm{MHz}\right) \delta$ : 10.51 (s, 1H), 8.86 (d, $J=7.6 \mathrm{~Hz}, 1 \mathrm{H}), 7.70$ (d, $J=7.2 \mathrm{~Hz}$, $1 \mathrm{H}), 7.46$ (t, $J=7.2 \mathrm{~Hz}, 1 \mathrm{H}), 7.27$ (t, $J=7.2 \mathrm{~Hz}, 1 \mathrm{H}), 7.09$ $(\mathrm{t}, J=7.6 \mathrm{~Hz}, 1 \mathrm{H}), 6.99 \sim 6.89(\mathrm{~m}, 3 \mathrm{H}), 3.96(\mathrm{t}, J=7.6 \mathrm{~Hz}$, 2H), $2.77(\mathrm{t}, J=7.2 \mathrm{~Hz}, 2 \mathrm{H}), 2.64(\mathrm{~s}, 4 \mathrm{H}), 1.81(\mathrm{~s}, 4 \mathrm{H}) ;{ }^{13} \mathrm{C}$ NMR $\left(\mathrm{CDCl}_{3}, 100 \mathrm{MHz}\right) \delta: 188.21,170.47,151.56$,
$141.14,139.23,136.83,129.12,125.56,125.18,122.54$, $121.52,121.22,119.98,111.82,107.99,106.49$, 54.35, 53.21, 39.15, 23.54; HRMS calcd for $\mathrm{C}_{22} \mathrm{H}_{22} \mathrm{~N}_{3} \mathrm{O}_{2}[\mathrm{M}+$ $\mathrm{H}]^{+}$360.1707, found 360.1707 .

$N^{1}$-[3-(1-哌啶)丙基]靛玉红(5e)：紫色固体，产率 72\%. m.p. 130 132 ${ }^{\circ} \mathrm{C} ;{ }^{1} \mathrm{H}$ NMR $\left(\mathrm{CDCl}_{3}, 400 \mathrm{MHz}\right) \delta$ : 10.52 (s, 1H), 8.87 (d, $J=6.4 \mathrm{~Hz}, 1 \mathrm{H}), 7.73$ (d, $J=5.6 \mathrm{~Hz}$, 1H), 7.49 (s, 1H), 7.29 (d, $J=6.0 \mathrm{~Hz}, 1 \mathrm{H}), 7.11$ (s, 1H), $7.01 \sim 6.95(\mathrm{~m}, 3 \mathrm{H}), 3.88(\mathrm{~s}, 2 \mathrm{H}), 2.50(\mathrm{~s}, 4 \mathrm{H}), 2.02(\mathrm{~s}$, $2 \mathrm{H}), 1.66(\mathrm{~s}, 4 \mathrm{H}), 1.46(\mathrm{~s}, 2 \mathrm{H}), 1.25(\mathrm{~s}, 2 \mathrm{H}) ;{ }^{13} \mathrm{C} \mathrm{NMR}$ $\left(\mathrm{CDCl}_{3}, 100 \mathrm{MHz}\right) \delta: 188.27,170.68,151.59,141.24$, $139.25,136.89,129.20,125.50,125.23,122.58,121.57$, $121.14,120.01,111.85,108.20,106.54,56.08,54.35$, 38.04, 29.69, 25.26, 24.76, 23.92; HRMS calcd for $\mathrm{C}_{24} \mathrm{H}_{26} \mathrm{~N}_{3} \mathrm{O}_{2}[\mathrm{M}+\mathrm{H}]^{+}$388.2020, found 388.2021.

\subsubsection{CCK-8 法测定目标产物的体外抗肿瘤活性}

以靛玉红和依托泊苷为阳性药，利用 CCK-8 法测 试目标产物分子对 HL-60 细胞的体外抑制活性. HL-60 细胞接种于 96 孔板中, 孵化 $24 \mathrm{~h}$. 根据组别设置加入含 有测试药物的培养基，并设立溶媒组、阴性对照组和阳

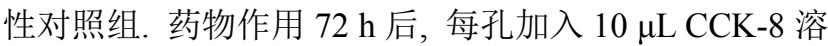
液，再孵化 $3 \mathrm{~h}$. 用酶标仪在 $450 \mathrm{~nm}$ 处测定每孔的吸光 度值(OD), 并计算抑制率. 实验结果用 SPSS17.0 计算 得到半数抑制浓度值 $\left(\mathrm{IC}_{50}\right)$.

\subsection{4 细胞周期检测}

将 HL-60 细胞接种到 6 孔板中, 孵化 $24 \mathrm{~h}$. 再根据 组别设置, 加入不同浓度的 $\mathbf{5 a}$ 培养基, 并设立阴性对照 组. 作用 $48 \mathrm{~h}$ 后, 收集细胞, 并进行离心, 再用体积分 数为 $70 \%$ 冰乙醇固定. 加 $100 \mu \mathrm{L}$ RNase A 孵化 $0.5 \mathrm{~h}$, 再加入 $400 \mu \mathrm{L}$ PI 进行染色. 最后, 用流式细胞仪检测 $488 \mathrm{~nm}$ 处苂光值.

\subsection{5 细胞凋亡检测}

将 HL-60 细胞接种到 6 孔板中，孵化 $24 \mathrm{~h}$. 再根据 组别设置，加入不同浓度的 $\mathbf{5} \mathbf{a}$ 培养基，并设立阴性对照 组. 药物作用 $48 \mathrm{~h}$ 后, 加入 $500 \mu \mathrm{L}$ Binding Buffer, 再分 别加入 $5 \mu \mathrm{L}$ Annexin V-APC 以及 $5 \mu \mathrm{L}$ 7-AAD, 放置 15 min. 最后，用流式细胞仪检测细胞凋亡情况.

\subsubsection{Hoechst 33342 染色}

HL-60 细胞与不同浓度 $\mathbf{5 a}$ 共同孵化 $24 \mathrm{~h}$ 后, 收集 细胞, 并浸入 $4 \%$ 的多聚甲醛固定液中 $0.5 \mathrm{~h}$. 再加入 Hoechst 33342 染色液，室温放置 $10 \mathrm{~min}$ 后，用苂光显微 镜观察细胞情况.

\subsection{7 蛋白质免疫印迹}

HL-60 细胞与不同浓度 $\mathbf{5 a}$ 共同孵化 $48 \mathrm{~h}$ 后，提取 
细胞中总蛋白, 方法同文献[28, 29]. 蛋白经变性处理 后，于质量分数为 $12 \%$ 的 SDS-PAGE 凝胶电泳分离转移 到 $\mathrm{NC}$ 膜膜上, 加入质量分数为 $5 \%$ 的脱脂奶粉的封闭 液封闭 $2 \mathrm{~h}$, 再加入 CDK1、CDK2 或 Cleaved Capase-3 一抗于 $4{ }^{\circ} \mathrm{C}$ 下捊化过夜. TBST 洗涤三次, 每次 $10 \mathrm{~min}$, 用相应的二抗室温孵化 $2 \mathrm{~h}, \mathrm{TBST}$ 洗涤三次, 每次 10 min. 最后, 用 ECL 化学发光试剂检测蛋白质印迹, 并 用 G-BOX ChemiXR5 凝胶成像分析系统成像.

辅助材料(Supporting Information) 目标产物的 ${ }^{1} \mathrm{H}$ $\mathrm{NMR},{ }^{13} \mathrm{C} \mathrm{NMR}, \mathrm{HRMS}$ 谱图. 这些材料可以免费从本 刊网站(http://sioc-journal.cn/)上下载.

\section{Referenes}

[1] Döhner, H.; Estey, E. H.; Amadori, S.; Appelbaum, F. R.; Büchner, T.; Burnett, A. K.; Dombret, H.; Fenaux, P.; Grimwade, D.; Larson, R. A.; Lo-Coco, F.; Naoe, T.; Niederwieser, D.; Ossenkoppele, G. J.; Sanz, M. A.; Sierra, J.; Tallman, M. S.; Löwenberg, B.; Bloomfield, C. D. Blood 2010, 115,453 .

[2] Ofran, Y.; Rowe J. M. Acta Haematol. 2014, 132, 292.

[3] Choi, T. Y.; Lee, M. S.; Ernst, E. Support. Care Cancer 2015, 23, 1819.

[4] Cai, J.; Damaraju, V. L.; Groulx, N.; Mowles, D.; Peng, Y.; Robins, M. J.; Cass, C. E.; Gros, P. Cancer Res. 2008, 68, 2349.

[5] Xiao, Z.; Hao, Y.; Liu, B.; Qian, L. Leukocyte Lymph. 2002, 43, 1763.

[6] Gaboriaud-Kolar, N.; Vougogiannopoulou, K.; Skaltsounis, A. L. Expert Opin. Ther. Patents 2015, 25, 583.

[7] Ponnusamy, K.; Petchiammal, C.; Mohankumar, R.; Hopper, W. J. Ethnopharmacol. 2010, 132, 349.

[8] Hsieh, W. L.; Lin, Y. K.; Tsai, C. N.; Wang, T. M.; Chen, T. Y.; Pang, J. H. S. J. Dermatol. Sci. 2012, 67, 140.

[9] Mak, N. K.; Leung, C. Y.; Wei, X. Y.; Shen, X. L.; Wong, R. N. S.; Leung, K. N.; Fung, M. C. Biochem. Pharmacol. 2004, 67, 167.

[10] Kunikata, T.; Tatefuji, T.; Aga, H.; Iwaki, K.; Ikeda, M.; Kurimoto, M. Eur. J. Pharmacol. 2000, 410, 93.

[11] Zhang, X.; Song, Y.; Wu, Y.; Dong, Y.; Lai, L.; Zhang, J.; Lu, B.; Dai, F.; He, L.; Liu, M.; Yi, Z. Int. J. Cancer 2011, 129, 2502.

[12] Suzuki, K.; Adachi, R.; Hirayama, A.; Watanabe, H.; Otani, S.;
Watanabe, Y.; Kasahara, T. Brit. J. Haematol. 2005, 130, 681.

[13] Gu, Y. C.; Li, G. L.; Yang, Y. P.; Fu, J. P.; Li, C. Z. Acta Pharm. Sinica 1989, 24, 629 (in Chinese).

(顾月翠，李国林，杨尧平，付建平，李成章，药学学报，1989, 24, 629.)

[14] Hsuan, S. L.; Chang, S. C.; Wang, S. Y.; Liao, T. L.; Jong, T. T.; Chien, M. S.; Lee, W. C.; Chen, S. S.; Liao, J. W. J. Ethnopharmacol. 2009, 123, 61 .

[15] Hoessel, R.; Leclerc, S.; Endicott, J. A.; Nobel, M. E. M.; Lawrie, A.; Tunnah, P.; Leost, M.; Damiens, E.; Marie, D.; Marko, D.; Niederberger, E.; Tang, W.; Eisenbrand, G.; Meijer, L. Nat. Cell Biol. $1999,1,60$.

[16] Vougogiannopoulou, K.; Ferandin. Y.; Bettayeb, K.; Myrianthopoulos, V.; Lozach, O.; Fan, Y.; Johnson, C. H.; Magiatis, P.; Skaltsounis, A. L.; Mikros, E.; Meijer, L. J. Med. Chem. 2008, 51, 6421.

[17] Song, J. H.; Lee, J. E.; Cho, K. M.; Park, S. H.; Kim, H. J.; Kim, Y. C.; Kim, T. S. Mol. Carcinog. 2016, 55, 611.

[18] Libnow, S.; Methling, K.; Hein, M.; Michalik, D.; Harms, M.; Wende, K.; Flemming, A.; Köckerling, M.; Reinke, H.; Bednarski, P. J.; Lalk, M.; Langer, P. Bioorg. Med. Chem. 2008, 16, 5570.

[19] Cheng, X.; Rasqué, P.; Vatter, S.; Merz, K. H.; Eisenbrand, G. Bioorg. Med. Chem. 2010, 18, 4509.

[20] Wang, T. C.; Wei, J. Z.; Guo, C. S.; Zhang, H. B.; Fan, H. X. Chin. Chem. Lett. 2010, 21, 1407.

[21] Wang, Z. H.; Li, W. Y.; Li, F. L.; Zhang, L.; Hua, W. Y.; Cheng, J. C.; Yao, Q. Z. Chin. Chem. Lett. 2009, $20,542$.

[22] Wang, Z.; Wang, Y.; Feng, M.; Tan, X.; Cheng, J.; Hua, W.; Yao, Q. Chin. J. Org. Chem. 2009, 29, 1606 (in Chinese). (王朝晖, 王越, 冯明声, 谭雪, 程景才, 华维一, 姚其正, 有机 化学, 2009, 29, 1606.)

[23] Wang, Z. H.; Dong, Y.; Wang, T.; Shang, M. H.; Hua, W. Y.; Yao, Q. Z. Chin. Chem. Lett. 2010, 21, 297.

[24] Cragg, G. M.; Grothaus, P. G.; Newman, D. J. Chem. Rev. 2009 109, 3012.

[25] Evan, G. I.; Vousden, K. H. Nature 2001, 411, 342.

[26] Fesik, S. W. Nat. Rev. Cancer 2005, 5, 876.

[27] Klöcka, C.; Jin, X.; Choi, K.; Khosla, C.; Madrid, P. B.; Spencer, S.; Raimundo, B. C.; Boardman, P.; Lanza, G.; Griffin, J. H. Bioorg. Med. Chem. Lett. 2011, 21, 2692.

[28] Zhang, L.; Zhang, Z.; Chen, F.; Chen, Y.; Lin, Y.; Wang, J. Eur. J. Med. Chem. 2016, 123, 226.

[29] Zhang, L.; Liu, L.; Zheng, C.; Wang, Y.; Nie, X.; Shi, D.; Chen, Y.; Wei, G.; Wang, J. Eur. J. Med. Chem. 2017, 131, 81.

(Li, L.; Fan, Y.) 